\title{
Photoexcited Triplet State Kinetics Studied by Electron Paramagnetic Resonance Spectroscopy
}

\author{
Christian Hintze, Ulrich E. Steiner, and Malte Drescher*[a]
}

Following the first evidence of the triplet character of the optically excited phosphorescent state of naphthalene by Hutchison and Mangum in 1958, ${ }^{[1]}$ electron paramagnetic resonance (EPR) spectroscopy has become widely used to study and understand the properties and kinetic characteristics of excited triplet states. This minireview gives an overview over EPR techniques based on continuous microwave methods using lock-in or direct detection as well as pulsed EPR methods with respect to their suitability for kinetic studies of excited triplet states. A short historical overview of the experimental and theoretical developments in this field of research as well as of the triplet systems studied, with a final focus on fullerenes, is given. This may help newcomers to the field as a guide to the relevant literature.

\section{Introduction}

In 1958, Hutchison and Mangum ${ }^{[1]}$ reported the first direct observation of the phosphorescent state of naphthalene by EPR, thus proving its triplet nature. About 10 years later, van der Waals and co-workers ${ }^{[2]}$ detected the phenomenon of optical spin polarization (OSP) in such systems, that is, the fact that the triplet spin sublevels were not created with initial Boltzmann distribution. This discovery marked the beginning of an era of intense studies on excited triplet states, EPR spectroscopy figuring most prominent among the methods to understand and characterize such states. ${ }^{[3]}$

Photoexcited triplet states have found practical applications in several areas, of which photosensitization ${ }^{[4]}$ may be the most important. Other significant fields are photovoltaics, ${ }^{[5]}$ organic electroluminescent devices ${ }^{[6]}$ and photon upconversion at low light intensities via triplet-triplet annihilation. ${ }^{[7]}$ In NMR spectroscopy, the high OSP of photoexcited triplet spins may be used to achieve proton spin polarization as high as $70 \%$ or more by dynamic nuclear polarization (DNP) ${ }^{[8,9]}$ Recently, the triplet state of porphyrin has been shown to be useful as a potential spin label for distance measurements in macromolecules within the nanometer range. ${ }^{[10,11]}$

Basically, OSP is due to unequal population/and or depopulation rates of the triplet substates. Therefore, in most of the applications mentioned, it is essential to know the respective kinetic parameters. During the years, various reviews have covered the progress in triplet state EPR, the first one having been given by Thomson in $1968,{ }^{[12]}$ which appeared shortly before the classical treatise on the spectroscopy of the triplet state by McGlynn, Azumi and Kinoshita. ${ }^{[13]}$ This early work essentially

[a] C. Hintze, Prof. Dr. U. E. Steiner, Prof. Dr. M. Drescher

Fachbereich Chemie

Universität Konstanz

78457 Konstanz (Germany)

Email:malte.drescher@unikonstanz.de refers to stationary continuous wave (CW-EPR) approaches. Later, time-resolved EPR (TR-EPR) techniques suitable for the investigation of short-lived paramagnetic transients were developed comprising direct detection (DD-EPR) and pulsed electron spin echo (ESE-EPR) methods. ${ }^{[14-17]}$ Studies of excited triplet states using ESE-EPR spectroscopy have been described in refs. [18, 19], for applications of DD-EPR cf. [20-22]. Many timeresolved triplet studies have been reported in the field of photosynthesis. ${ }^{[23-28]}$ In his review of 2001, a personal account given on the occasion of his Zavoisky award lecture, Van der Waals highlights the historical perspectives of the EPR of excited triplet states. ${ }^{[3]}$ Another technique for the study of excited triplets is optically detected magnetic resonance (ODMR), ${ }^{[29,30]}$ which, as an optical method, is extremely sensitive, such that triplet state kinetics can be studied even for single molecules. ${ }^{[31]}$ In this concise review, however, we restrict ourselves to EPR techniques based on microwave detection.

The review is organized as follows. In Section 2, we present an outline of the structure of excited triplet state EPR spectra and the relevant kinetic equations for the triplet substates, including the solution for special conditions. In the subsequent sections we characterize the three time-resolved EPR techniques, conveniently applied for resolving population and depopulation kinetics as well as spin relaxation in photoexcited triplet states. These sections describe CW-EPR (Section 3.1), DD-EPR (Section 3.2) and ESE-EPR (Section 4) and applications thereof. A special section of applications is devoted to Fullerenes (Section 5). Finally, Section 6 presents the summary and conclusions.

\section{Excited Triplet-State Kinetics}

Triplet-state kinetics is embedded in a sequence of electronic excitation and conversion processes usually represented in a Jablonski diagram ${ }^{[32]}$ as shown in Figure 1. Generally, for most molecules the ground state is diamagnetic, corresponding to 
an electronic singlet state $\mathrm{S}_{0}$. On absorption of a photon, it is excited to a higher singlet state, because of conservation of spin for electric dipole allowed processes. For the sake of simplicity, we will restrict our consideration to the first excited singlet state $\mathrm{S}_{1}$.

Due to the Franck-Condon principle, electronic excitation is usually coupled with vibrational excitation. Excess vibrational energy is usually dissipated within $<10^{-12} \mathrm{~s}$ by vibrational relaxation. Since the following processes are usually slower by orders of magnitude, they proceed from the vibrationless excited $\mathrm{S}_{1}$ state. These processes comprise emission of a photon

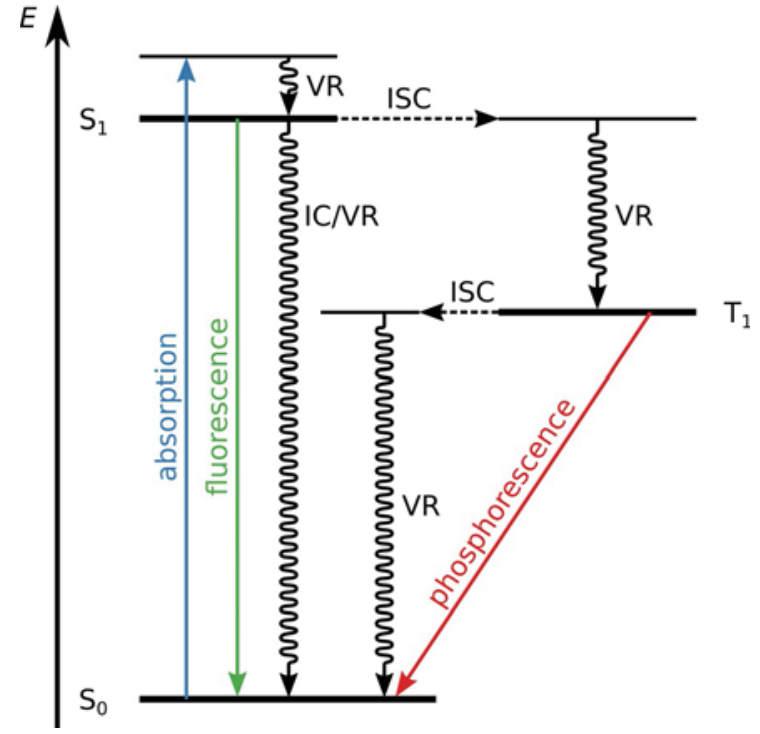

Figure 1. Jablonski diagram. Radiative transitions are colored corresponding to their energy, decreasing from blue to red. Intersystem crossing is indicat ed by dotted arrows. Wavy lines represent vibrational relaxation (VR) and in ternal conversion (IC) processes. Energy levels drawn with thin lines repre sent higher vibrational states of the respective lower electronic level.

(fluorescence, typically $10^{-8} \mathrm{~s}$ ), radiationless transition to a higher vibrational level of the ground state (internal conversion, IC, typically $10^{-9} \mathrm{~s}$ ) and radiationless transition, including a change of multiplicity to the paramagnetic triplet state with subsequent vibrational relaxation (intersystem crossing, ISC, typically $10^{-9} \mathrm{~s}$ ). The triplet state may be deactivated by emission (phosphorescence, typically $10^{-2} \mathrm{~s}$ ) and by ISC to the singlet ground state (typically $10^{-3} \mathrm{~s}$ at cryogenic temperatures).

A triplet state comprises three spin sublevels, which, unless the geometry is spherical or of cubic or icosahedral symmetry, differ in energy even in zero magnetic field. This energy splitting is called zero-field splitting (ZFS) ${ }^{[13]}$ and is due to magnetic dipolar interaction between the unpaired spins and/or to spinorbit coupling. Even in aromatic organic molecules, the latter may become significant through the presence of heavy atom substituents or hetero atoms introducing $n \pi^{*}$ excited states. ${ }^{[33,34]}$ When dealing with the triplet state quantum theoretically, the ZFS is conveniently described by a spin Hamiltonian $\hat{H}_{D}=\vec{S} D \vec{S}$, where $\vec{S}$ is the spin vector operator and $D$ the zero field splitting tensor. In a coordinate system where $D$ is diagonal, the ZFS Hamiltonian can be written in the form [Eq. (1)]:

$\hat{H}_{\mathrm{D}}=D\left(\begin{array}{ll}\hat{S}_{z}^{2} & \frac{1}{3} \hat{S}^{2}\end{array}\right)+E\left(\begin{array}{ll}\hat{S}_{x}^{2} & \hat{S}_{y}^{2}\end{array}\right)$

Here, $D$ and $E$ are the so-called ZFS parameters. For axial symmetry, the parameter $E$ is zero. For cubic or higher symmetry, both ZFS parameters are zero. The ZFS is not visible in the energy scale underlying the Jablonski diagram in Figure 1, because the energy differences involved in electronic transitions correspond to frequencies of the order of some hundred $\mathrm{THz}$, whereas $D$, the larger of the two ZFS parameters, is typically on the order of a few $\mathrm{GHz}$. 
The ZFS parameters define the energetic splitting and ordering of the triplet sublevels in zero field. In the eigenstates of the ZFS Hamiltonian, denoted $T_{x}, T_{y}$ and $T_{z}$, the spin is oriented perpendicular to the pertinent axes. Their energies, with respect to their center of gravity, are [Eq. (2)]:

$E_{x}=\frac{1}{3} D \quad E$
$E_{y}=\frac{1}{3} D+E$
$E_{z}=\frac{2}{3} D$

The standard convention for assigning the labels $x, y$ and $z$ to the molecular axes is such that $T_{z}$ is the level with the largest separation from the center of gravity, $T_{x}$ with the second largest separation, and $T_{y}$ is in the middle. This convention implies that $1 / 3 \leq E / D \leq 0$, that is, the signs of $D$ and $E$ are always different, and the absolute value of $E$ is bounded by $1 /$ 3 of the absolute value of $D .^{[35]}$ With $D>0, T_{z}$ has the lowest energy, with $D<0$ the highest. With $E<0, \mathrm{~T}_{x}$ is higher than $\mathrm{T}_{y}$ with $E>0$ it is vice versa (Figure 2).

Applying an external magnetic field, the triplet levels are further split by the Zeeman interaction represented by the Zeeman Hamiltonian $\hat{H}_{z}=\mu_{\mathrm{B}} \vec{B}_{0} g \hat{\vec{S}}$ with $\mu_{\mathrm{B}}$ Bohr's magneton, $\vec{B}_{0}$ the magnetic field and $g$ the $g$-tensor. The energy splitting as a function of the magnetic field are shown in Figure 2 for $D>$ 0 and for $D<0$ for all three canonical orientations, that is, with one of the molecular axes parallel to the external magnetic field. The eigenvalues of the spin Hamiltonian are given by
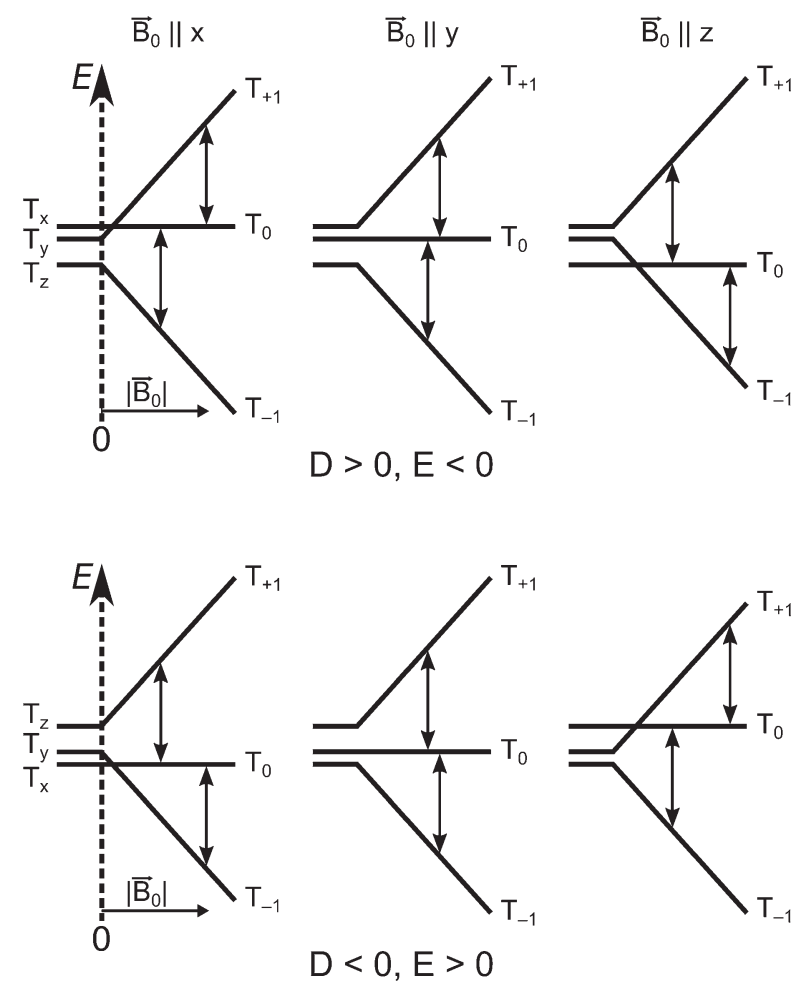

Figure 2. Magnetic field dependence of the spin energy levels for the three canonical orientations for $D>0$ (top) and $D<0$ (bottom). The $|0\rangle \leftrightarrow|1\rangle$ and $|0\rangle \leftrightarrow|+1\rangle$ transitions for each canonical orientation $i=x, y, z$ shall be denoted by $1 i$ and $2 i$, respectively. Then, the six $\Delta m= \pm 1$ transitions in the direction from low field to high field are $(2 z)(1 x)(1 y)(2 y)(2 x)(1 z)$ for $D>0$ and $(1 z)(2 x)(2 y)(1 y)(1 x)(2 z)$ for $D<0$
Equations (3): $:^{[36]}$

$$
\begin{aligned}
& \vec{B}_{0} \| x: E_{ \pm 1}=\frac{1}{2}\left(\frac{D}{3} \quad E\right) \pm \sqrt{\frac{1}{4}}(D+E)^{2}+\left(g \mu_{\mathrm{B}} B_{0}\right)^{2}, E_{0}=\frac{1}{3} D \quad E
\end{aligned}
$$

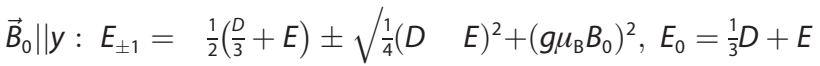

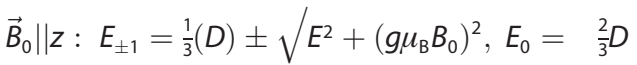

and, in the limit of high field, that is, $g \mu_{\mathrm{B}} B_{0} \gg D^{*}=\sqrt{ } D^{2}+3 E^{2}$, the eigenfunctions by Equation (4):

$$
\begin{aligned}
& \vec{B}_{0}|| x:| \pm 1\rangle=\left(\frac{1}{\sqrt{2}}\right)\left(\left|T_{y}\right\rangle \pm i\left|T_{z}\right\rangle\right),|0\rangle=\left|T_{x}\right\rangle \\
& \vec{B}_{0}|| y:| \pm 1\rangle=\left(\frac{1}{\sqrt{2}}\right)\left(\left|T_{z}\right\rangle \pm i\left|T_{x}\right\rangle\right),|0\rangle=\left|T_{y}\right\rangle \\
& \vec{B}_{0}|| z:| \pm 1\rangle=\left(\frac{1}{\sqrt{2}}\right)\left(\left|T_{x}\right\rangle \pm i\left|T_{y}\right\rangle\right),|0\rangle=\left|T_{z}\right\rangle
\end{aligned}
$$

For the eigenstates in case of low and intermediate values of the magnetic field $B_{0}$, please see Ref. [36].

When considering an isotropic distribution of triplet state orientations, for example, in powders or solid solutions, a spectral distribution of resonances results as shown in Figure $3 \mathrm{~A}$. Here the canonical orientations stand out as positive steps or maxima along the field axis, if we consider a Boltzmann population of the levels. The ordering of the canonical transitions as shown in Figure 3 depends on the sign of $D$ and follows the pertinent sequence given in the caption of Figure 2. In turn, the ZFS parameters can be determined from the canonical spectral positions of an experimental powder spectrum.

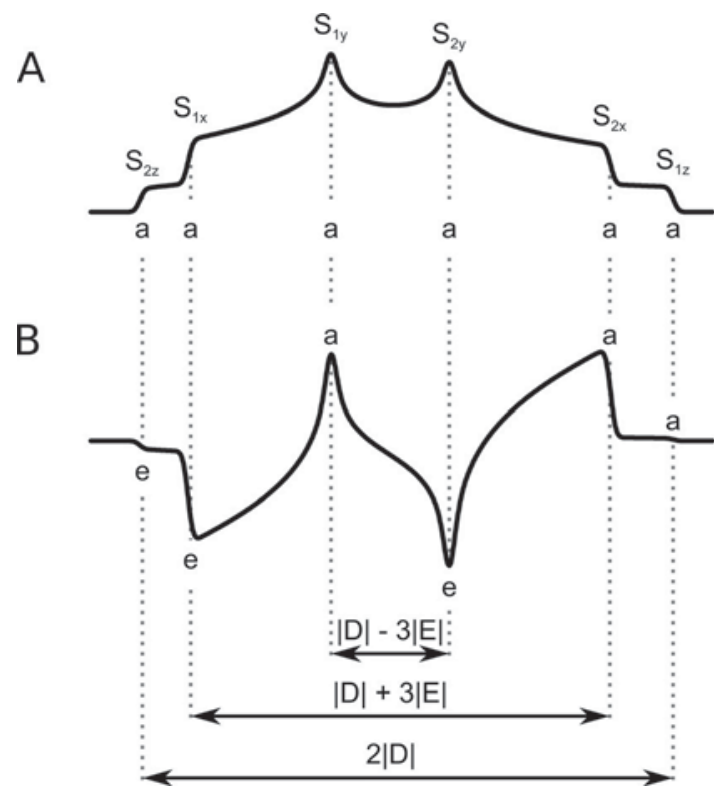

Figure 3. EPR spectra of randomly oriented triplets ("powder spectra" simu lated with EasySpin, a MATLAB toolbox). ${ }^{[3]}$ A) In thermal equilibrium, all sig nals are in absorption, indicated by letter "a". B) Spin polarized spectrum with some signals in absorption (a) and some in emission (e). The signal in tensities $S_{p i}(p=1,2$ and $i=x, y, z)$ are labeled according to the definitions (case $D>0$ ) given in the caption of Figure 2 . 
Immediately after their formation by ISC, the three triplet sublevels are usually not populated according to a Boltzmann distribution. This phenomenon is called optical spin polarization (OSP) and causes enhanced EPR absorption, but also emission, in which latter case the steps in the powder spectrum become negative and maxima turn into minima (Figure $3 \mathrm{~B}$ ).

Optically polarized triplets relax towards Boltzmann equilibrium by spin-lattice relaxation (SRL) while each of the triplet sublevels individually decays to the ground state $S_{0}$ either via phosphorescence or via ISC or a combination of both of these types of processes. The details are shown in Figure 4, the individual substate population rates in zero field being denoted by $s_{i}$ and their decay rates by $k_{i}(i=x, y, z)$.

The individual population and depopulation rates of the triplet substates are determined by the symmetry rules of spinorbit coupling (SOC) that are not necessarily selective for exact eigenstates of the triplet spin Hamiltonian, but for states to be represented as coherent superpositions of the energy eigenstates. ${ }^{[3,38]}$ However, in most cases of direct spectroscopy of excited triplet states, the coherences decay faster than detectable and it is appropriate to consider incoherent population and depopulation of the eigenstates of the effective spin Hamiltonian.

Using the transformation relations of the ZFS substates with the eigenstates in a general magnetic field, the rate constants in zero field and non-zero magnetic fields can be related to each other by the squares of the pertinent coefficients. Thus, for example, in high fields along the canonical directions the result is given by Equations (5) and (6):

$\vec{B}_{0} \| x: s_{ \pm 1}=\frac{1}{2}\left(s_{y}+s_{z}\right), s_{0}=s_{x}$

$\vec{B}_{0} \| y: s_{ \pm 1}=\frac{1}{2}\left(s_{x}+s_{z}\right), s_{0}=s_{y}$

$\vec{B}_{0} \| z: s_{ \pm 1}=\frac{1}{2}\left(s_{x}+s_{y}\right), s_{0}=s_{z}$

$\vec{B}_{0} \| x: k_{ \pm 1}=\frac{1}{2}\left(k_{y}+k_{z}\right), k_{0}=k_{x}$

$\vec{B}_{0} \| y: k_{ \pm 1}=\frac{1}{2}\left(k_{x}+k_{z}\right), k_{0}=k_{y}$

$\vec{B}_{0} \| z: k_{ \pm 1}=\frac{1}{2}\left(k_{x}+k_{y}\right), k_{0}=k_{z}$

The rate constants $w_{p}(p=1,2,3)$ specify spin-lattice relaxation (SLR) among the triplet substates. For them, a simple transformation between zero-field and in-field values does not exist, since SLR depends on the energy separation between the levels. The SLR rates for up and down process between the same pair of levels are related by the Boltzmann factor $e_{p}=\exp \left(\frac{|\Delta E|}{k_{b} T}\right)$.

Based on the kinetic scheme in Figure 4, the relaxation of an optically spin polarized triplet spectrum into Boltzmann equilibrium and/or its decay due to transitions to the singlet ground state can be treated quantitatively. Denoting the individual high-field triplet sublevel populations by $N_{i}(i=1,0,+1)$, the pertinent rate equations following from Figure 4 are [Eq. (7)]:

$\left(\begin{array}{c}\dot{N}_{+1} \\ \dot{N}_{0} \\ \dot{N}_{-1}\end{array}\right)=\left(\begin{array}{ccc}\left(k_{+1}+w_{2}+w_{3}\right) & w_{2} e_{2} & w_{3} e_{3} \\ w_{2} & \left(k_{0}+w_{1}+w_{2} e_{2}\right) & w_{1} e_{1} \\ w_{3} & w_{1} & \left(k_{-1}+w_{1} e_{1}+w_{3} e_{3}\right)\end{array}\right)\left(\begin{array}{c}N_{+1} \\ N_{0} \\ N_{-1}\end{array}\right)$

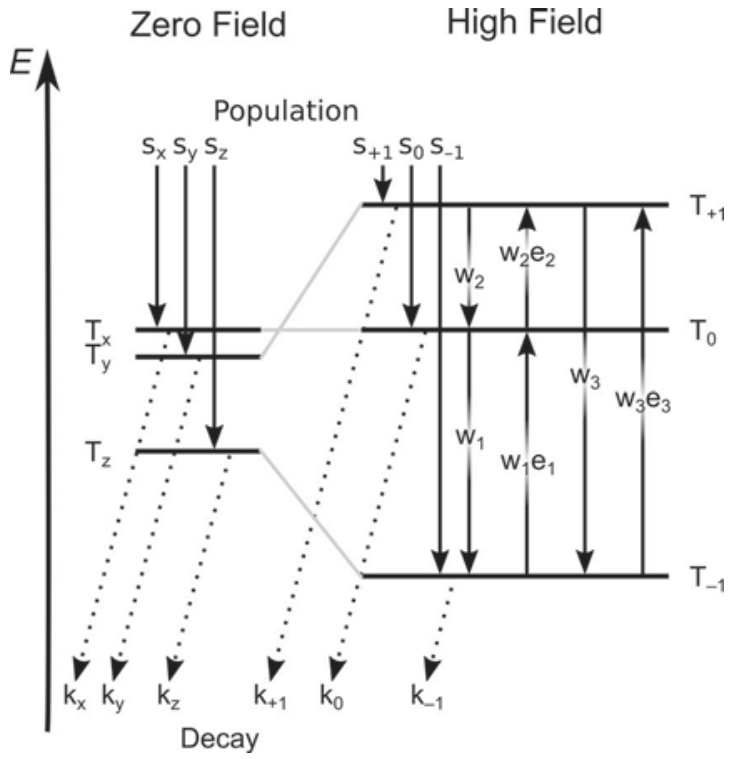

Figure 4. Assignment of transition rate constants for triplet sublevel scheme in zero and high field. The dotted arrows indicate combined radiationless (ISC) and radiative (phosphorescence) decay.

The initial conditions for the populations, that is, their values immediately after photoexcitation of the triplet, are determined by the population rates $s_{i}$ [Eq. (8)]:

$\frac{N_{i}}{\sum_{j} N_{j}}=\frac{s_{i}}{\sum_{j} s_{j}}$

In the high-field limit, the initial populations of the upper and lower triplet sublevels are equal, $N_{+1}(t=0)=N_{-1}(t=0)$ [Eq. (5)]. The system of differential equations cannot be solved analytically for general values of the rate parameters. In the following we consider the solutions for simple cases of slow $\left(w_{p} \ll k_{i}\right)$ and fast SLR $\left(w_{p} \gg k_{i}\right)$.

\subsection{Slow Spin Lattice Relaxation/Fast Triplet Decay}

In case of slow SLR $\left(w_{p} \ll k_{i}\right)$, Equation (8) simplifies considerably, to yield Equation (9):

$$
\left(\begin{array}{c}
\dot{N}_{+1} \\
\dot{N}_{0} \\
\dot{N}_{-1}
\end{array}\right)=\left(\begin{array}{ccc}
k_{+1} & 0 & 0 \\
0 & k_{0} & 0 \\
0 & 0 & k_{-1}
\end{array}\right)\left(\begin{array}{c}
N_{+1} \\
N_{0} \\
N_{-1}
\end{array}\right)
$$

The three equations are completely decoupled. Each triplet sublevel decays individually by a first-order rate process.

\subsection{Fast Spin Lattice Relaxation in High-Temperature Limit}

If $\operatorname{SLR}$ is fast $\left(w_{p} \gg k_{i}\right)$, the system first equilibrates into the Boltzmann distribution and then decays slowly into the singlet ground state. For the relaxation kinetics the rate equations are 


$$
\left(\begin{array}{c}
\dot{N}_{+1} \\
\dot{N}_{0} \\
\dot{N}_{-1}
\end{array}\right)=\left(\begin{array}{ccc}
\left(w_{2}+w_{3}\right) & w_{2} e_{2} & w_{3} e_{3} \\
w_{2} & \left(w_{1}+w_{2} e_{2}\right) & w_{1} e_{1} \\
w_{3} & w_{1} & \left(w_{1} e_{1}+w_{3} e_{3}\right)
\end{array}\right)\left(\begin{array}{c}
N_{+1} \\
N_{0} \\
N_{-1}
\end{array}\right)
$$

In the high-temperature limit, the Boltzmann factors approach the value of 1 . We may also neglect double quantum transitions of SLR, meaning that $w_{3}=0$, and we may use the approximation $w_{1}=w_{2}=w$. Then Equation (10) simplifies to Equation (11):

$$
\left(\begin{array}{c}
\dot{N}_{+1} \\
\dot{N}_{0} \\
\dot{N}_{-1}
\end{array}\right)=\left(\begin{array}{ccc}
w & w & 0 \\
w & 2 w & w \\
0 & w & w
\end{array}\right)\left(\begin{array}{c}
N_{+1} \\
N_{0} \\
N_{-1}
\end{array}\right)
$$

This can easily be solved to yield Equation (12):

$$
\left(\begin{array}{c}
\dot{N}_{+1} \\
\dot{N}_{0} \\
\dot{N}_{-1}
\end{array}\right)=\left(\begin{array}{ccc}
1 & 1 & 1 \\
2 & 0 & 1 \\
1 & 1 & 1
\end{array}\right)\left(\begin{array}{c}
C_{1} e^{-3 w t} \\
C_{2} e^{-3 w t} \\
C_{3}
\end{array}\right)
$$

Taking into account that under high field conditions $N_{+1}(0)=$ $N_{-1}(0)$, it follows that $C_{2}=0$. Since the EPR signal intensities are proportional to population differences we conclude that these are proportional to [Eq. (13)]:

$$
\begin{array}{ll}
S_{1} \propto N_{1}^{-}=N_{0} & N_{-1}=3 C_{1} e^{-3 w t} \\
S_{2} \propto N_{2}^{-}=N_{+1} & N_{0}=+3 C_{1} e^{-3 w t}
\end{array}
$$

that is, they are equal in amplitude but of opposite sign, and they decay monoexponentially with a rate constant of $3 w$, equivalent to the inverse of the SLR time $T_{1}$. For long times $t>$ $(15 w)^{-1}$ the signals decay to zero due to the approximation of infinite temperature. For many practical applications, the approximations used so far are not adequate. Therefore, some less restrictive treatments are reviewed next.

\subsection{Less Restrictive Approximations}

The first quantitative treatment of triplet state kinetics was presented by Schwoerer and Sixl. ${ }^{[39]}$ They solved the kinetic equations under the approximation of high-field and fast relaxation, however further taking into account the Boltzmann factors and a microwave pumping term since their measurements were carried out under (weak) continuous microwave irradiation. They also derived the conditions for the reversion of absorption or emission on switching off the optical pumping (cf. Section 3.1).

On a similar basis of approximation, Levanon and Vega simulated the growing in and decay of the CW-EPR signal upon switching on and off the optical pumping. They also treated the CW-EPR response under conditions of sinusoidal modulation of the pumping light intensity. ${ }^{[40]}$
While the latter authors assumed relaxation between all triplet sublevels as equal, Winscom, ${ }^{[41]}$ who treated the triplet kinetics under continuous microwave irradiation and various conditions of periodic changes of the pumping light intensity, provided perturbational solutions for the case of different relaxation rate constants for different values of $|\Delta m|$. A generalization of the Levanon-Vega treatment avoiding the high-field approximation has been provided by Hiromitsu and Kevan. ${ }^{[42]}$

The kinetic equations valid in the case of CW-EPR are simplified in two respects when turning to experiments utilizing electron ESE-EPR in combination with a short ns-laser pulse for optically pumping the triplet. Since the time interval of creation of the triplets in this case is short on the EPR time scale, one does not have to treat the explicit population kinetics, but can include the population rates in the initial populations of the triplet sublevel [Eq. (8)]. Furthermore, the time evolution of the substate populations is not complicated by the absorption of microwave power. These advantages of ESE EPR have been exploited by Doetschman and Botter for determining all individual SLR constants between the sublevels of triplet diphenylmethylene, a molecule with a stable triplet ground state, in which case there was not even a necessity for optical pumping. ${ }^{[43]}$

Seidel, Mehring and Stehlik ${ }^{[44]}$ employed a somewhat extended version of the theory presented in Section 2.2 to analyze their experimental results from ESE-EPR experiments with the acridine triplet doped into fluorine. They did not resort to the limit $w_{p} \gg k_{i}$ from the beginning, but applied it at the stage of the general solution of the eigenvalues of the kinetic matrix, thus keeping a term that still depends on the $k_{i}$. In the notation of the present paper, the resulting kinetics is given by Equation (14):

$$
N_{1}^{-}(t)=N_{2}^{-}(t)=\left[N_{2}^{-}(0)+\alpha N^{+}(0)\right] e^{-3 w t} \quad \alpha N^{+}(0) e^{-k t}
$$

where $N^{+}=N_{0}+N_{ \pm 1}$, the average decay rate $k=\frac{1}{3}\left(\begin{array}{ll}2 k_{ \pm 1} & k_{0}\end{array}\right)$ and the kinetic anisotropy factor $\alpha$ is defined as Equation (15):

$\alpha=\frac{\frac{1}{3}\left(k_{ \pm 1} \quad k_{0}\right)}{3 w}$

The second exponential describes the slow EPR echo signal decay after the initial fast decay, determined by the SLR time $T_{1} \approx(3 w)^{-1}$ has reached a quasi-stationary fraction $\alpha$ of the remaining population. In case the decay is isotropic $\alpha=0$. Then it would appear that decay rates cannot be determined from the EPR kinetics. But of course, there is still a tail resulting from the triplet population in Boltzmann equilibrium given by Equation (16):

$N_{\mathrm{B}}^{-}(t)=N_{\mathrm{B}}^{-}(0) e^{-k t}$

from which the rate constant of triplet decay can be determined. 


\subsection{Radical Spin Polarization from Polarized Triplets in Solution}

Although it is beyond the scope of this short review, it may be of interest and shall be briefly mentioned that the treatment of triplet sublevel kinetics is also an essential issue in dealing with spin-polarized radicals (chemically induced dynamic electron spin polarization, CIDEP) ${ }^{[45]}$ originating from the so-called triplet mechanism, ${ }^{[46,47]}$ according to which spin polarization induced by SOC effects during population and/or depopulation in excited triplet-state precursors is transferred to radicals formed by chemical reactions in the triplet state such as homolytic bond cleavage, hydrogen atom transfer or electron transfer. In those scenarios, formation, reaction and decay of the triplets usually takes place in liquid solutions and the dynamic treatment of triplet kinetics has to take into account the effect of rotational diffusion. ${ }^{[48]}$ Polarization effects in liquid solution can be due not only to spin sublevel selective population of triplets but also to selective depopulation, ${ }^{[49]}$ if the latter processes are fast enough, which requires systems with strong SOC. ${ }^{[49-51]}$ Due to fast dephasing and spin relaxation caused by rotational diffusion, the triplet spin polarization is efficiently transferred to the subsequent radicals only if the radical formation reactions are fast. Hence, due to the short time scale of the triplet kinetics, coherence effects must be taken into account, which necessitates a quantum kinetic treatment based on the spin density matrix with an appropriate master equation. ${ }^{[48,49]}$

\section{Continuous Microwave Irradiation Methods}

\subsection{Lock-In Detection}

The first experimental evidence of OSP of triplets came from phosphorescence life time measurements by de Groot et al. ${ }^{[52]}$ the first direct detection of OSP of triplets by EPR was reported by Schwoerer and Six ${ }^{[39]}$ in an experiment using continuous illumination and CW-EPR. In order to observe OSP as a steadystate phenomenon under continuous illumination, SLR has to be slow compared to the triplet lifetime, so that the polarization is maintained during the whole life time of the triplet. Therefore, these experiments were carried out at liquid helium temperature.

In these first time-resolved EPR experiments, the change of EPR line intensities was recorded after switching on or off the light (cf. Figure 5). In this way, the kinetics of several triplet species in various hosts, ${ }^{[39,53]}$ the effect of deuteration and the aspect of spin conservation in triplet-triplet energy transfer between host and guest molecules have been studied. Similar types of studies were performed by Clarke. ${ }^{[54]}$

Improvements of the time resolution of the CW-EPR method by employing light modulation, either by chopping or through a series of flash lamp pulses, with phase sensitive signal detection opened access also to the kinetics of shorter lived triplets at liquid nitrogen temperature ${ }^{[55,56]}$ with particular interest in the triplet states of chlorophylls in glassy solution. ${ }^{[57,58]}$

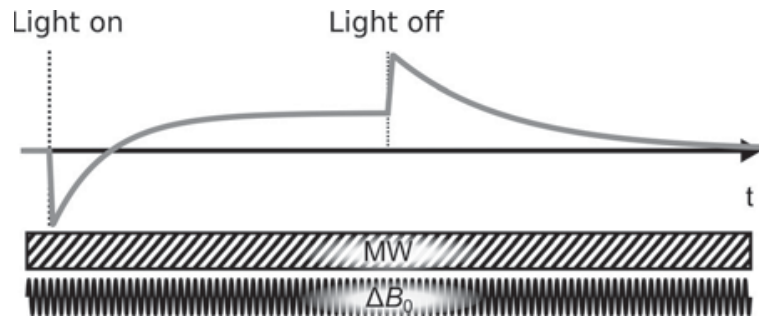

Figure 5. Scheme of a CW EPR experiment with lock in detection and light pulses switched on and off. The signal trace is drawn in gray. The time scale is on the order of $\mathrm{ms}$ to $\mathrm{s}$.

With a standard modulation frequency of the magnetic field of $100 \mathrm{kHz}$, lock-in detectors allowed for time resolutions down to $20 \mu \mathrm{s} .{ }^{[26]}$ Increasing the modulation frequency to 1$2 \mathrm{MHz}$ it was possible to reduce the time resolution to $1 \mu \mathrm{s}$, however with the drawback of a reduced SNR due to the small modulation amplitudes available at such high modulation frequencies. $^{[26]}$ Using $200 \mathrm{kHz}$ modulation and modifying the lock-in detector by replacing low-pass filtering by signal averaging, De Jager and Van Wijk managed to achieve a time resolution of less than $1 \mu s^{[59]}$ which, in the case of broad EPR lines, showed better SNR than the high frequency modulation method.

In CW-EPR methods, separating the rate constants of triplet decay from those of SLR may be challenging, if both are of similar order of magnitude. As was first demonstrated by Wolfe ${ }^{[60]}$ switching microwave power from a high saturating level to a low monitoring level, while maintaining the sample under continuous illumination by light, the time domain response of the EPR signal is determined by SLR only.

\subsection{Direct Detection}

Historically, the first transient magnetic resonance experiment was the NMR transient nutation experiment described by Torrey in $1949 .{ }^{[61]}$ Since the advent of Fourier transform techniques, transient nutation experiments are no longer of importance in modern NMR. In EPR, however, this technique is still of interest for the investigation of transient paramagnetic states. ${ }^{[20-22]}$

In a transient nutation experiment, the magnetization is observed under the condition of continuous application of an oscillatory resonant magnetic field $B_{1}$ orthogonal to the static magnetic field $B_{0}$. If the resonant interaction can be approximated by a two-level scheme, the Bloch vector description of motion of the magnetization is applicable. ${ }^{[21]}$ After switching on the resonant field, the magnetization vector starts a precession around the $B_{1}$ direction in the rotating frame. By $90^{\circ}$ out of phase detection a signal oscillating with the Rabi frequency $\omega_{R}=g \mu_{\mathrm{B}} B_{1}$ is observed, which is damped by the spin relaxation time $T_{2}$.

The first application of this technique in EPR is due to Wolfe ${ }^{[60]}$ In his experiments, the microwave was applied as a sequence of high power pulses superimposed on a low level basis of microwave power while keeping the sample under 
constant optical pumping producing the triplet states. If the SLR relaxation rates $w_{i}$ are much larger than the triplet decay rate, the relaxation of the signal after the saturating microwave pulses is determined by the SLR time constants $w_{i}$. By this method, it could be shown for a series of host-guest systems, that the temperature dependence of the SLR rates is characteristic of a combination of Raman and direct processes. The method has been also applied by Schwoerer et al. to investigate SLR processes in different mixed crystals. ${ }^{[62]}$

Soon it appeared that the use of intense laser pulses for optical pumping that are short in comparison to the triplet decay and spin kinetics, offers the possibility to perform DD-EPR without high power microwave pulses at all. This method was first applied to the CIDEP of photochemically generated radicals ${ }^{[17,63,64]}$ and later to triplets. ${ }^{[21,65]}$ The experimental principle is schematically shown in Figure 6. If the spin levels of the light-induced species are spin polarized, any standard CW-EPR spectrometer will be sufficient to perform DD-EPR.

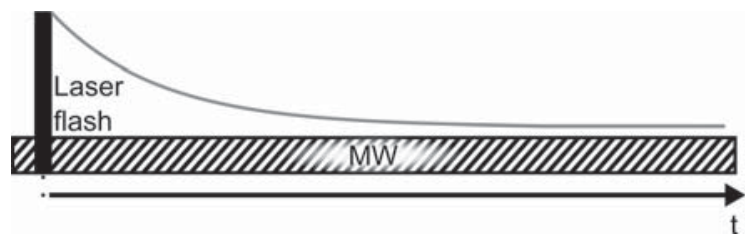

Figure 6. Scheme of the DD EPR experiment where the transient signal is re corded without magnetic field modulation after a laser flash. The observed signal is drawn in green. The time scale is on the order of $\mathrm{ns}$ to ms.

In general, the Bloch equations applied to pulse generated spin-polarized species may yield oscillatory or non-oscillatory solutions. At high microwave power, one observes the underdamped oscillatory behavior described above. In that case the transient signals decay with the spin-spin relaxation time $T_{2}$. At low microwave powers, the signals become overdamped, that is, they decay monotonically. In that case the exponential decay rate represents the SLR time $T_{1}$.

\section{Pulsed Microwave Techniques}

With the advantages of high time resolution in the nanosecond range, high sensitivity and ready availability of pulsed spectrometers, ESE-EPR is best suited to study excited triplet states. For this purpose, the basic Hahn echo pulse sequence (cf. Figure 7) is sufficient. In CW-EPR, one has to take care not to distort the kinetic data by too high microwave power, because of disturbing additional microwave stimulated transitions. Due to this restriction, the sensitivity of the CW technique is reduced. By contrast, in ESE-EPR one can apply optimal combinations of microwave pulse length and microwave power.

The first ESE-EPR experiment on photoexcited triplet states was reported by van der Waals and co-workers ${ }^{[66]}$ on quinoxaline- $d_{6}$ and naphthalene- $d_{8}$ in durene host crystals at $1.2 \mathrm{~K}$. In analogy to the first CW-EPR work on triplet kinetics by Schwoerer and Sixl, ${ }^{[39]}$ they showed the development of the ESE in-

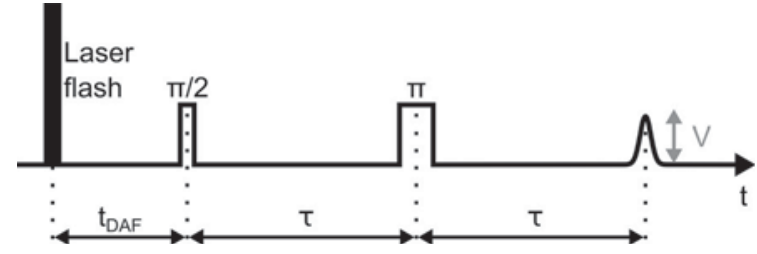

Figure 7. Hahn echo sequence used in ESE EPR. In order to assess the triplet state kinetics, the ESE intensity $V$ is detected as a function of time delay $t_{\mathrm{DAF}}$ between the laser flash and the first microwave pulse. The delay $\tau$ between the pulses is optimized for maximum electron spin echo intensity $V$. The time scale is on the order of ns to $\mathrm{s}$.

tensity upon closing the shutter of a CW excitation light. At the low temperature of the experiment, SLR can be neglected and the observed signal decay rates reflect the triplet life time. Due to the applied shutter technique of switching the CW light, the time resolution of this experiment was comparable to that of the mentioned CW-EPR one, that is, on the order of tens of ms. For the study of short-lived triplets of pentacene in naphthalene at $1.2 \mathrm{~K}$, the time resolution was significantly enhanced down to some $\mu$ s by using laser flash excitation. ${ }^{[67]}$ In this case, the triplet kinetics is determined by varying the time delay $t_{\mathrm{DAF}}$ between laser flash and the first microwave pulse.

\section{Representative Application: Fullerenes}

As soon as the theoretical and methodological basis for the investigations of photoexcited triplet state kinetics with EPR had been established, these techniques were readily applied in several areas of science. Comprehensive reviews of EPR applications within various specific fields exist (see the Introduction), and are beyond the scope of this review. As a final representative example, however, demonstrating the potential of EPR spectroscopy to reveal important information and essential understanding of electronic structure and molecular dynamics the case of fullerenes, an important new class of compounds, shall be briefly reviewed.

After fullerenes became available on a laboratory scale in $1990,{ }^{[68]}$ all kinds of spectroscopic investigations started immediately. ${ }^{[69]}$ Optical experiments revealed a very high triplet yield of both $C_{60}$ and $C_{70} \cdot{ }^{[0,71]}$ Phosphorescence of $C_{60}$ is very weak, ${ }^{[72]}$ but moderate in the case of $C_{70} \cdot{ }^{[73]}$ The first EPR detection of excited triplet states of $\mathrm{C}_{60}$ and $\mathrm{C}_{70}$ were reported by Wasielesky and co-workers. ${ }^{[73]}$ Working in an organic glass at 5-9 K, the EPR spectra were recorded by CW-EPR with fast light modulation and the triplet lifetimes by DD-EPR.

In the case of $C_{60}$, EPR measurements by Bennati et al. ${ }^{[74]}$ as well as ODMR measurements by Lane et al. ${ }^{[75]}$ revealed that as the temperature increases there is a second EPR signal with smaller ZFS parameters overlaying the low-temperature spectrum and gaining more and more weight at higher temperature. The activation energy to form the second triplet species was determined as $17 \mathrm{meV}^{[74]}$ In ref.[76] it is concluded from ODMR experiments that the second triplet was "extrinsic", that is, not belonging to a pure $\mathrm{C}_{60}$ species. 
In the ground state, the geometry of the $\mathrm{C}_{60}$ molecule represents an ideal icosahedron (symmetry group $I_{h}$ ). In the excited state such a conformation is not stable due to the Jahn-Teller effect. The fact that the observed ZFS parameters are not zero is indicative of a lowering of symmetry upon photoexcitation. As shown theoretically by Wang et al., ${ }^{[77]}$ in the lowest excited state the molecule is stabilized by a distortion along any of the $C_{5}$ axes thus leading to a shape of $D_{5 d}$ symmetry. Since there are six such axes, the excited state potential surface exhibits six distinct equivalent minima. Transitions between the corresponding conformations represent pseudo-rotations of the molecule. EPR and ODMR spectroscopy does indeed provide evidence of such processes. From a simulation of the spectral ODMR line shape, Wei et al. derived a hopping frequency between the $\mathrm{C}_{60}$ pseudorotamers of $2 \mathrm{MHz}$ at $2 \mathrm{~K}$ and of $10 \mathrm{MHz}$ at $40 \mathrm{~K}$ with a linear temperature dependence in between, formally in line with a one-phonon mechanism.

The very narrow EPR line of only $0.14 \mathrm{G}$ linewidth observed at room temperature was interpreted by Closs et al. ${ }^{[78]}$ as due to a motional narrowing effect based on very rapid interchange of magnetic axes by pseudorotation, converting the degenerate Jahn-Teller states into each other. At lower temperatures of 203 and $253 \mathrm{~K}$, they were able to observe the triplet decay and relaxation using DD-EPR. The interpretation of the narrow high-temperature line as being due to a motionally narrowed triplet spectrum was confirmed in joint work by the groups of van Willigen and Linschitz ${ }^{[79]}$ who also observed electron transfer quenching of the $\mathrm{C}_{60}$ triplet by the electron donor tri-p-tolylamine (TTA) confirming independent results obtained by flash photolysis. ${ }^{[00]}$ These experiments clearly showed that the interpretation of the narrow EPR line at RT as due to a radical (either $\mathrm{C}_{60}{ }^{-}$or $\mathrm{e}^{-}$) advocated in other work ${ }^{[81,82]}$ was not correct.

The structure of $C_{70}$ in its ground state is less symmetric than of $\mathrm{C}_{60}$. It adopts the shape of a prolate ellipsoid (rugby ball) with a five-fold rotational symmetry along its long axis. ${ }^{[83]}$ In its excited triplet state, there is also a small Jahn-Teller distortion disturbing the axial symmetry, as evidenced by a nonvanishing, albeit small, ZFS parameter $\mathrm{E}\left(0.00069 \mathrm{~cm}^{-1}\right)$ which is of the same size as for $\mathrm{C}_{60}{ }^{[73]}$ Closs et al. ${ }^{[78]}$ were the first to investigate the temperature dependence of the $C_{70}$ EPR spectrum. Different from $\mathrm{C}_{60}$, an EPR spectrum is not detectable in liquid solution above $180 \mathrm{~K}$. The spectrum exhibits a sharp structure below $100 \mathrm{~K}$. When raising the temperature, the lines broaden and the whole spectral width decreases to end up in a single broad line of $8.8 \mathrm{G}$ linewidth. Then the line, while still narrowing, gets weaker and disappears at about $180 \mathrm{~K}$. Closs et al. ascribed this behavior to the relaxational effect of proper rotation of the molecule.

Terazima et al. found that the sublevel decay rates and the SLR time for $C_{70}$ showed considerable anisotropy. ${ }^{[84]}$ In a more detailed, temperature dependent DD-EPR study between ca. 30 and $77 \mathrm{~K}_{1}^{[85]}$ they analyzed the spectral change in terms of a model with spectral diffusion due to pseudorotational jumps between the five axial Jahn-Teller minima. The model, which fits the spectral changes quite well, yields hopping rates between $6 \times 10^{4} \mathrm{~s}^{-1}$ and $3 \times 10^{6} \mathrm{~s}^{-1}$ in the investigated tempera- ture range, leading to an activation energy of $E_{\mathrm{A}} \approx 250 \mathrm{~cm}^{-1}$ for the jumps. The model is, however, at variance with conclusions from a later investigation by Dauw et al., ${ }^{[8]}$ who studied the $C_{70}$ triplet at $1.2 \mathrm{~K}$ using the ESE-EPR technique at X-band and $\mathrm{W}$-band. The high field experiments allowed determination of the sublevel specific population and depopulation rate, showing that $75 \%$ of the initial population goes to $T_{z}$ and that the decay of that level is about three times as fast as from $T_{x}$ or $T_{y}$. Depending on the matrix, the SLR relaxation time was found to be 10 to $20 \mathrm{~ms}$. The signal decays at various spectral positions were only explicable in terms of the $|\Delta m|=2$ process. Such an order of magnitude is in accord with the general Redfield expression for $T_{1}$ if one assumes a modulation of the ZFS parameter $E$ by pseudorotational axial hopping at a frequency of about $1 \mathrm{MHz}$, a value identical with the $T_{2}$ time evaluated from the ESE decay.

Very recently, Uvarov et al. ${ }^{[87]}$ reinvestigated the temperature dependent SLR behavior of ${ }^{3} C_{70}$ using ESE inversion recovery. Whereas the time constants of ESE inversion recovery were similar to the rate of spectral change observed by Terazima et al. ${ }^{[85]}$ their interpretation as SLR time is at variance with the spectral diffusion model. The SLR rates observed by Uvarov et al. obeyed an Arrhenius behavior with an activation energy of ca. $170 \mathrm{~cm}^{-1}$. Therefore, the SLR mechanism was assigned to be of the Orbach-Aminov type, involving transition to a close-by higher electronic state with an energy separation equal to the activation energy. The fact that the same SLR times were obtained for X-band and Q-band is another strong argument for the Orbach-Aminov mechanism.

\section{Conclusions}

In this minireview, a short historical overview of the experimental and theoretical developments of EPR spectroscopy applied to kinetic studies of excited triplet states as well as of the triplet systems studied, with a final focus on fullerenes, has been given which may help newcomers to the field as a guide to the relevant literature.

In practical applications of photoexcited triplets such as DNP, triplet doublet double electron-electron resonance (DEER) or laser-induced magnetic dipole spectroscopy (LaserIMD), knowledge of triplet state kinetics is essential for choosing the right triplet system for a given experiment. For example, in DNP, triplet spin polarization has to persist long enough to achieve high polarization transfer. On the other hand, the triplet system should return to the singlet ground state within the experimental repetition time, so that enough chromophores are available for excitation during the next cycle. There are rich data on triplet quantum yields, life times, ZFS parameters, and optical spectra in the literature (cf. [88]), however no comprehensive overview of EPR relevant data on triplet kinetics exists. Therefore, Table 1 summarizes those triplet systems and their conditions of investigation, for which kinetic parameters are to be found in the literature mentioned in this review. 


\begin{tabular}{|c|c|c|c|c|c|}
\hline \multirow{2}{*}{$\begin{array}{l}\text { Chromophore } \\
\text { Acenapthene }\end{array}$} & \multirow{2}{*}{$\begin{array}{l}\text { Matrix or Solvent } \\
\text { Fluorene }\end{array}$} & \multirow{2}{*}{$\begin{array}{l}\text { Temperature } \\
\text { in } \mathrm{K} \\
4.2\end{array}$} & \multirow{2}{*}{$\begin{array}{l}\text { Triplet Decay } \\
\text { Rate(s) } \\
x\end{array}$} & \multicolumn{2}{|c|}{$\begin{array}{l}\text { SLR } \\
\text { rate(s) }\end{array}$} \\
\hline & & & & {$[x]$} & {$[53,89]$} \\
\hline 1 Acetylcyclohexene & Trifluorethanol & 77 & $x$ & & {$[90]$} \\
\hline Anthracene & Fluorene & 4.2 & $x$ & {$[x]$} & {$[53,89]$} \\
\hline Anthracene d10 & Fluorene & $4.2 / 230$ & $x$ & {$[x]$} & [91] \\
\hline Anthracene d10 & Toluene $\mathrm{d} 8$ & 50 & $x$ & $x$ & [92] \\
\hline Anthracene (X Traps) & Anthracene + Phenazine & $1.6 / 4.2$ & $x$ & {$[x]$} & [91] \\
\hline Benzophenone & Toluene & 30 & & $x$ & [93] \\
\hline Benzophenone in $\beta$ Cyclodextrine & Water/Glycerole & 30 & & $x$ & [93] \\
\hline Boron phthalocyanine & Toluene & 163263 & $x$ & $x$ & [94] \\
\hline $\mathrm{C} 60$ & Benzonitrile & 9210 & $x$ & $x$ & [74] \\
\hline $\mathrm{C} 60$ & Methylcyclohexane & 3 & $x$ & & [84] \\
\hline $\mathrm{C} 60$ & Methylcyclohexane & $8 / 203 / 253$ & $x$ & $x$ & [78] \\
\hline $\mathrm{C} 60$ & Toluene & 9 & $x$ & & [73] \\
\hline $\mathrm{C} 60$ & Toluene & 253 & & $x$ & [81] \\
\hline $\mathrm{C} 60$ & Toluene & 150380 & $x$ & $x$ & [96] \\
\hline $\mathrm{C} 60$ & Toluene & 180340 & $x$ & $x$ & {$[96]$} \\
\hline $\mathrm{C} 60$ & Toluene, E7 liquid crystal & 8177 & $x$ & & [95] \\
\hline C70 & Decalin & 30100 & & $x$ & [87] \\
\hline $\mathrm{C} 70$ & Decalin/Cyclohexane & 1.2 & $x$ & $x$ & {$[86]$} \\
\hline C70 & Methylcyclohexane & 3 & $x$ & & [84] \\
\hline $\mathrm{C} 70$ & Toluene & 1.2 & $x$ & $x$ & {$[86]$} \\
\hline C70 & Toluene & 9 & $x$ & & [73] \\
\hline Chlorophyll a & n Octane & 85 & $x$ & $x$ & [58] \\
\hline Chlorophyll a/b & MTHF; Toluene & 95 & $x$ & & [97] \\
\hline Chlorophyll b & Ethanol & 77 & $x$ & & [57] \\
\hline Chlorophyll b & MTHF & 77 & $x$ & & {$[57]$} \\
\hline 2 Cyclohexenone & Trifluorethanol & 77 & $x$ & & [90] \\
\hline 2 Cyclopentenone & Trifluorethanol & 77 & $x$ & & [90] \\
\hline 9,10 Diazaphenanthrene & Biphenyl; Fluorene & 3 & $x$ & & [99] \\
\hline 3,6 Dichloropyridazine & $p$ Dibromobenzene & 3180 & $x$ & & [100] \\
\hline Fluorene (X Traps) & Fluorene + Carbazol & 4.2 & $x$ & {$[x]$} & {$[53,89]$} \\
\hline Fluorene (X Traps) & Fluorene + Dibenzothiophene & 4.2 & $x$ & {$[x]$} & {$[53,89]$} \\
\hline 2 Methyl Naphthalene & Napthalene & 4.2 & $x$ & {$[x]$} & {$[53,89]$} \\
\hline Naphthalene & Biphenyl & $1.3 / 2.1 / 4.2$ & $(x)$ & $x$ & {$[60]$} \\
\hline Naphthalene & Durene & $1.3 / 2.1 / 4.2$ & $(x)$ & $x$ & {$[60]$} \\
\hline Naphthalene & Naphthalene d8 & 4.2 & $x$ & {$[x]$} & {$[53,101]$} \\
\hline Naphthalene & Naphthalene d8 & 0.54 .2 & & $x$ & [62] \\
\hline Naphthalene d8 & Biphenyl & $1.3 / 2.1 / 4.2$ & $(x)$ & $x$ & {$[60]$} \\
\hline Naphthalene $\mathrm{d} 8$ & Durene & $1.3 / 2.1 / 4.2$ & $(x)$ & $x$ & {$[60]$} \\
\hline Naphthalene (X Traps) & Napthalene + Benzothiophene & 4.2 & $x$ & {$[x]$} & {$[53,89]$} \\
\hline Naphthalene (X Traps) & Napthalene + Chinoxaline & 1.6 & $x$ & {$[x]$} & {$[53,89]$} \\
\hline P 700 & Water/Glycerole & 1.2 & $x$ & {$[x]$} & [102] \\
\hline P 700 & Water/Sorbitol & 10100 & & $x$ & [103] \\
\hline P 700 & Water/Triton X; Water/LDS & 5 & $x$ & {$[x]$} & [104] \\
\hline P 840 & Water/Glycerole & 5 & $x$ & & [105] \\
\hline Pentacene & Benzoic acid & $103 / 178 / 298$ & $x$ & $x$ & [106] \\
\hline Pentacene & Naphthalene & 1.2 & $x$ & & [67] \\
\hline Pentacene & $p$ Terphenyl & 298 & $x$ & $x$ & [107] \\
\hline Pentacene $\mathrm{d} 14$ & $p$ Terphenyl & 298 & $x$ & $x$ & [107] \\
\hline Phenanthrene & Fluorene & 4.2 & $x$ & {$[x]$} & {$[53,89]$} \\
\hline Phenazine & Fluorene & $1.6 / 4.2 / 230$ & $x$ & {$[x]$} & [91] \\
\hline Phenazine & Fluorene & 4.2220 & $x$ & $x$ & [108] \\
\hline Pyrene & Fluorene & 4.2 & $x$ & {$[x]$} & {$[53,89]$} \\
\hline Pyrene d10 & Fluorene & $1.6 / 230$ & $x$ & {$[x]$} & [91] \\
\hline Pyridazine & Ethanol & 3 & $x$ & & [109] \\
\hline Pyridazine & Ethanol & 377 & $x$ & & [100] \\
\hline Quinoxaline & Durene & 2.6 & & $x$ & [110] \\
\hline Quinoxaline & Durene & 2.6 & & $x$ & [111] \\
\hline
\end{tabular}




\begin{tabular}{|c|c|c|c|c|c|}
\hline Chromophore & Matrix or Solvent & $\begin{array}{l}\text { Temperature } \\
\text { in } \mathrm{K}\end{array}$ & $\begin{array}{l}\text { Triplet Decay } \\
\text { Rate(s) }\end{array}$ & $\begin{array}{l}\text { SLR } \\
\text { rate(s) }\end{array}$ & \\
\hline Quinoxaline & Durene & $1.3 / 2.1 / 4.2$ & $(x)$ & $x$ & {$[60]$} \\
\hline Quinoxaline & Naphthalene & 2.6 & & $x$ & {$[111]$} \\
\hline Quinoxaline & Naphthalene d8 & 2.6 & & $x$ & [111] \\
\hline Quinoxaline & Napthalene d8 & 2.6 & & $x$ & [110] \\
\hline Quinoxaline & Napthalene d8 & 4.2 & $x$ & {$[x]$} & {$[53,89]$} \\
\hline Quinoxaline d6 & Durene & 1.2 & $x$ & & {$[66]$} \\
\hline $\begin{array}{l}\text { reaction center of Rhodopseudomonas } \\
\text { sphaeroides }\end{array}$ & Water/Triton & 4.2 & $x$ & $x$ & [112] \\
\hline $\begin{array}{l}\text { reaction center of Rhodopseudomonas } \\
\text { sphaeroides }\end{array}$ & Water/Triton & 1560 & $x$ & & [113] \\
\hline $\begin{array}{l}\text { reaction center of Rhodopseudomonas vir } \\
\text { idis }\end{array}$ & Water/LDAO & 40100 & $x$ & & [114] \\
\hline self trapped exciton & $\mathrm{AgCl}$ & 1.12 .0 & $x$ & $(x)$ & [115] \\
\hline Tetra ( $m$ fluorophenyl)porphin & Ether/Ethanol( d1) & 1.74 .2 & $x$ & & {$[117]$} \\
\hline Tetramethyl /Tetraphenylporphyrin Dimers & Methyltetrahydrofuran/Toluene & 110 & & $x$ & [118] \\
\hline Tetramethylpyrazine & Durene & $1.3 / 2.1 / 4.2$ & $(x)$ & $x$ & {$[60]$} \\
\hline trans Stilbene & Diphenylacetylene & $250 \mid 5$ & $x$ & $x$ & [119] \\
\hline trans Stilbene & $\begin{array}{l}\text { Isopentane/3 Methylpentane; EPA; } 3 \text { Methylpen } \\
\text { tane; } 1 \text { Pentanol }\end{array}$ & 77 & $x$ & & [120] \\
\hline
\end{tabular}

\section{Acknowledgements}

This work was financially supported by the DFG (DR 743/10-1).

Keywords: electron paramagnetic resonance - epr spectroscopy $\cdot$ kinetics $\cdot$ relaxation $\cdot$ triplet states

[1] C. A. J. Hutchison, B. W. Mangum, J. Chem. Phys. 1958, 29, 952953.

[2] M. S. de Groot, I. A. M. Hesselmann, J. H. van der Waals, Mol. Phys. 1967, 12, 259264.

[3] J. H. van der Waals, Appl. Magn. Reson. 2001, 20, 545561.

[4] J. Zhao, W. Wu, J. Sun, S. Guo, Chem. Soc. Rev. 2013, 42, 53235351.

[5] N. J. Thompson, M. W. B. Wilson, D. N. Congreve, P. R. Brown, J. M. Scherer, T. S. Bischof, M. Wu, N. Geva, M. Welborn, T. V. Voorhis, V. Bu lović, M. G. Bawendi, M. A. Baldo, Nat. Mater. 2014, 13, 10391043.

[6] M. A. Baldo, D. F. O'Brien, Y. You, A. Shoustikov, S. Sibley, M. E. Thomp son, S. R. Forrest, Nature 1998, 395, 151154.

[7] T. N. Singh Rachford, F. N. Castellano, Coord. Chem. Rev. 2010, 254, 25602573.

[8] A. Henstra, T. S. Lin, J. Schmidt, W. T. Wenckebach, Chem. Phys. Lett 1990, 165, 610

[9] T. R. Eichhorn, N. Niketic, B. van den Brandt, U. Filges, T. Panzner, E. Rantsiou, W. T. Wenckebach, P. Hautle, Nucl. Instrum. Methods Phys. Res. Sect. A 2014, 754, 1014.

[10] M. Di Valentin, M. Albertini, E. Zurlo, M. Gobbo, D. Carbonera, J. Am. Chem. Soc. 2014, 136, 65826585.

[11] C. Hintze, D. Bücker, S. D. Köhler, G. Jeschke, M. Drescher, J. Phys. Chem. Lett. 2016, 22042209.

[12] C. Thomson, Q. Rev. 1968, 22, 4574.

[13] S. P. McGlynn, T. Azumi, M. Kinoshita, Molecular Spectroscopy of the Triplet State, Prentice Hall International, Hemel Hemptead, 1969.

[14] S. I. Weissman, Annu. Rev. Phys. Chem. 1982, 33, 301318.

[15] A. D. Trifunac, R. G. Lawler, D. M. Bartels, M. C. Thurnauer, Prog. React. Kinet. 1986, 14, 43156.

[16] K. A. McLauchlan in Time resolved EPR (Ed.: A. J. Hoff), Elsevier, Amster dam, 1989, pp. 345369

[17] D. Stehlik, K. Möbius, Annu. Rev. Phys. Chem. 1997, 48, 745784.

[18] J. R. Norris, M. C. Thurnauer, M. K. Bowman in Advances in Biological and Medical Physics, Vol. 17 (Eds.: J. H. Lawrence, J. W. Gofman, T. L. Hayes), Academic Press, New York, 1980, pp. 365416

[19] T. S. Lin, Chem. Rev. 1984, 84, 115.
[20] N. Hirota, S. Yamauchi, M. Terazima, Rev. Chem. Intermed. 1987, 8, 189 205.

[21] D. Stehlik, C. Bock, M. C. Thurnauer in Advanced EPR (Ed. A. J. Hoff), Elsevier, Amsterdam, 1989, pp. 371403.

[22] N. Hirota, S. Yamauchi, J. Photochem. Photobiol. C 2003, 4, 109124.

[23] H. Levanon, J. R. Norris, Chem. Rev. 1978, 78, 185198.

[24] A. J. Hoff, Phys. Rep. 1979, 54, 75200.

[25] M. C. Thurnauer, Rev. Chem. Intermed. 1979, 3, 197230.

[26] A. J. Hoff, Q. Rev. Biophys. 1984, 17, 153282.

[27] D. E. Budil, M. C. Thurnauer, Biochim. Biophys. Acta 1991, 1057, 141.

[28] H. Levanon, K. Möbius, Annu. Rev. Biophys. Biomol. Struct. 1997, 26, 495540.

[29] M. A. El Sayed, Annu. Rev. Phys. Chem. 1975, 26, 235258.

[30] D. Carbonera, Photosynth. Res. 2009, 102, 403414.

[31] R. Brown, J. Wrachtrup, M. Orrit, J. Bernard, C. V. Borczyskowski, J. Chem. Phys. 1994, 100, 71827191.

[32] J. R. Lakowicz, Principles of Fluorescence Spectroscopy, Springer, Boston, 2006.

[33] M. A. El Sayed, J. Chem. Phys. 1963, 38, 28342838.

[34] S. K. Lower, M. A. El Sayed, Chem. Rev. 1966, 66, 199241.

[35] C. P. J. Poole, H. A. Farach, J. Chem. Phys. 1974, 61, 22202221.

[36] A. Carrington, A. D. McLachlan, Introduction to Magnetic Resonance, Chapman and Hall, London, 1979.

[37] S. Stoll, A. Schweiger, J. Magn. Reson. 2006, 178, 4255.

[38] U. E. Steiner, T. Ulrich, Chem. Rev. 1989, 89, 51147.

[39] M. Schwoerer, H. Slxl, Z. Naturforsch. A 1969, 24, 952967.

[40] H. Levanon, S. Vega, J. Chem. Phys. 1974, 61, 22652274.

[41] C. J. Winscom, Z. Naturforsch. A 1975, 30, 571582.

[42] I. Hiromitsu, L. Kevan, J. Chem. Phys. 1988, 88, 691695.

[43] D. C. Doetschman, B. J. Botter, J. Magn. Reson. 1981, 44, 348354.

[44] H. Sèidel, M. Mehring, D. Stehlik, Chem. Phys. Lett. 1984, 104, 552559.

[45] L. T. Muus, P. W. Atkins, K. A. McLauchlan, J. B. Pedersen in Chemically induced magnetic polarization, D. Reidel, Dordrecht, 1977.

[46] S. K. Wong, D. A. Hutchinson, J. K. S. Wan, J. Chem. Phys. 1973, 58, 985989.

[47] U. E. Steiner, Ber. Bunsen Ges. 1981, 85, 228

[48] P. W. Atkins, G. T. Evans, Mol. Phys. 1974, 27, 16331644.

[49] A. Katsuki, Y. Kobori, S. Tero Kubota, S. Milikisyants, H. Paul, U. E. Stein er, Mol. Phys. 2002, 100, 12451259.

[50] S. Milikisyants, A. Katsuki, U. E. Steiner, H. Paul, Mol. Phys. 2002, 100, 12151224.

[51] Y. Sakaguchi, H. Hayashi, J. Phys. Chem. A 2004, 108, 34213429. 
[52] M. S. de Groot, I. A. M. Hesselmann, J. Schmidt, J. H. Vanderwa, Mol. Phys. 1968, 15, 1736.

[53] H. Sixl, Thesis, Universität Stuttgart, 1971

[54] R. H. Clarke, Chem. Phys. Lett. 1971, 12, 157160.

[55] S. I. Weissman, H. Levanon, J. Am. Chem. Soc. 1971, 93, 43094310.

[56] H. Levanon, Chem. Phys. Lett. 1971, 9, 257259.

[57] J. F. Kleibeuker, T. J. Schaafsma, Chem. Phys. Lett. 1974, 29, 116122.

[58] H. Levanon, A. Scherz, Chem. Phys. Lett. 1975, 31, 119124.

[59] P. A. de Jager, F. G. H. van Wijk, Rev. Sci. Instrum. 1987, 58, 735741.

[60] J. P. Wolfe, Chem. Phys. Lett. 1971, 10, 212218.

[61] H. C. Torrey, Phys. Rev. 1949, 76, 10591068.

[62] M. Schwoerer, U. Konzelmann, D. Kilpper, Chem. Phys. Lett. 1972, 13, 272277.

[63] R. W. Fessenden, J. Chem. Phys. 1973, 58, 24892500.

[64] P. W. Atkins, A. J. Dobbs, K. A. McLauchlan, Chem. Phys. Lett. 1974, 25, 105107.

[65] J. S. Leigh, P. L. Dutton, Biochim. Biophys. Acta Bioenerg. 1974, 357, 67 77.

[66] B. J. Botter, D. C. Doetschman, J. Schmidt, J. H. Vanderwaals, Mol. Phys. $1975,30,609620$.

[67] A. J. Van Strien, J. Schmidt, Chem. Phys. Lett. 1980, 70, 513517.

[68] W. Krätschmer, L. D. Lamb, K. Fostiropoulos, D. R. Huffman, Nature $1990,347,354358$.

[69] J. Shinar, Z. V. Vardeny, Z. H. Kafafi in Optical and Electronic Properties of Fullerenes and Fullerene Based Materials, Marcel Dekker, New York, 2000.

[70] J. W. Arbogast, A. P. Darmanyan, C. S. Foote, Y. Rubin, F. N. Diederich, M. M. Alvarez, S. J. ANnz, R. L. Whetten, J. Phys. Chem. 1991, 95, 1112.

[71] J. W. Arbogast, C. S. Foote, J. Am. Chem. Soc. 1991, 113, 88868889.

[72] D. J. van den Heuvel, I. Y. Chan, E. J. J. Groenen, J. Schmidt, G. Meijer, Chem. Phys. Lett. 1994, 231, 111118.

[73] M. R. Wasielewski, M. P. O'Neil, K. R. Lykke, M. J. Pellin, D. M. Gruen, J. Am. Chem. Soc. 1991, 113, 27742776.

[74] M. Bennati, A. Grupp, M. Mehring, K. P. Dinse, J. Fink, Chem. Phys. Lett. 1992, 200, 440444.

[75] P. A. Lane, L. S. Swanson, Q. X. Ni, J. Shinar, J. P. Engel, T. J. Barton, L. Jones, Phys. Rev. Lett. 1992, 68, 887890.

[76] X. Wei, Z. V. Vardeny, Phys. Rev. B 1995, 52, R2317 R2320.

[77] W. Z. Wang, C. L. Wang, Z. B. Su, L. Yu, Phys. Rev. Lett. 1994, 72, 3550 3553.

[78] G. L. Closs, P. Gautam, D. Zhang, P. J. Krusic, S. A. Hill, E. Wasserman, J. Phys. Chem. 1992, 96, 52285231.

[79] C. A. Steren, P. R. Levstein, H. van Willigen, H. Linschitz, L. Biczok, Chem. Phys. Lett. 1993, 204, 2328.

[80] L. Biczok, H. Linschitz, R. I. Walter, Chem. Phys. Lett. 1992, 195, 339 346.

[81] H. Levanon, V. Meiklyar, A. Michaeli, S. Michaeli, A. Regev, J. Phys. Chem. 1992, 96, 61286131.

[82] M. Ruebsam, K. P. Dinse, M. Plueschau, J. Fink, W. Kraetschmer, K. Fos tiropoulos, J. Am. Chem. Soc. 1992, 114, 1005910061.

[83] D. R. McKenzie, C. A. Davis, D. J. H. Cockayne, D. A. Muller, A. M. Vassal lo, Nature 1992, 355, 622624.

[84] M. Terazima, N. Hirota, H. Shinohara, Y. Saito, Chem. Phys. Lett. 1992, 195, 333338.

[85] M. Terazima, K. Sakurada, N. Hirota, H. Shinohara, Y. Saito, J. Phys. Chem. 1993, 97, 54475450.

[86] X. L. R. Dauw, O. G. Poluektov, J. B. M. Warntjes, M. V. Bronsveld, E. J. J. Groenen, J. Phys. Chem. A 1998, 102, 30783082.
[87] M. N. Uvarov, J. Behrends, L. V. Kulik, J. Chem. Phys. 2015, 143, 244314 [88] M. Montalti, A. Credi, L. Prodi, M. T. Gandolfi, Handbook of Photochem istry, 3rd ed., CRC, Boca Raton, 2006.

[89] H. Sixl, M. Schwoerer, Z. Naturforsch. A 1970, 25, 13831394.

[90] S. Yamauchi, N. Hirota, J. Higuchi, J. Phys. Chem. 1988, 92, 21292133.

[91] J. Gromer, H. Sixl, H. C. Wolf, Chem. Phys. Lett. 1972, 12, 574578.

[92] S. D. Köhler, S. Höfel, M. Drescher, Mol. Phys. 2013, 111, 29082913.

[93] O. Krumkacheva, M. Tanabe, S. Yamauchi, M. Fedin, S. R. A. Marque, E. Bagryanskaya, Appl. Magn. Reson. 2012, 42, 2940.

[94] S. Yamauchi, A. Takahashi, Y. Iwasaki, M. Unno, Y. Ohba, J. Higuchi, A. Blank, H. Levanon, J. Phys. Chem. A 2003, 107, 14781485.

[95] A. Regev, D. Gamliel, V. Meiklyar, S. Michaeli, H. Levanon, J. Phys. Chem. 1993, 97, 36713679.

[96] C. A. Steren, H. van Willigen, K. P. Dinse, J. Phys. Chem. 1994, 98, 7464 7469.

[97] R. H. Clarke, R. E. Connors, T. J. Schaafsma, J. F. Kleibeuker, R. J. Platen kamp, J. Am. Chem. Soc. 1976, 98, 36743677.

[98] M. Terazima, J. Chem. Phys. 1987, 87, 37893795

[99] M. Terazima, Chem. Phys. Lett. 1987, 140, 610.

[100] M. Terazima, S. Yamauchi, N. Hirota, J. Chem. Phys. 1986, 84, 3679 3687.

[101] M. Schwoerer, H. Sixl, Chem. Phys. Lett. 1968, 2, 1419.

[102] P. Setif, J. P. Quaegebeur, P. Mathis, Biochim. Biophys. Acta Bioenerg. $1982,681,345353$

[103] K. A. Rose, A. Bearden, Biochim. Biophys. Acta Bioenerg. 1980, 593, 342352.

[104] P. Gast, T. Swarthoff, F. C. R. Ebskamp, A. J. Hoff, Biochim. Biophys. Acta Bioenerg. 1983, 722, 163175.

[105] T. Swarthoff, P. Gast, A. J. Hoff, FEBS Lett. 1981, 127, 8386.

[106] H. L. Yu, T. S. Lin, S. I. Weissman, D. J. Sloop, J. Chem. Phys. 1984, 80, 102107.

[107] D. J. Sloop, H. L. Yu, T. S. Lin, S. I. Weissman, J. Chem. Phys. 1981, 75, 37463757.

[108] S. S. Kim, S. I. Weissman, Chem. Phys. 1978, 27, 2126.

[109] M. Terazima, S. Yamauchi, N. Hirota, Chem. Phys. Lett. 1985, 120, 321 326.

[110] U. Konzelmann, M. Schwoerer, Chem. Phys. Lett. 1973, 18, 143146.

[111] U. Konzelmann, D. Kilpper, M. Schwoerer, Z. Naturforsch. A 1975, 30, 754770.

[112] P. Gast, A. J. Hoff, FEBS Lett. 1978, 85, 183188.

[113] A. J. Hoff, I. I. Proskuryakov, Chem. Phys. Lett. 1985, 115, 303310.

[114] F. G. H. van Wijk, T. J. Schaafsma, Biochim. Biophys. Acta Bioenerg. 1988, 936, 236248

[115] O. G. Poluektov, M. C. J. M. Donckers, P. G. Baranov, J. Schmidt, Phys. Rev. B 1993, 47, 1022610234

[116] A. J. Van Strien, J. Schmidt, Chem. Phys. Lett. 1982, 86, 203208.

[117] A. M. P. Goncalves, R. P. Burgner, Chem. Phys. Lett. 1977, 46, 488492.

[118] P. Jaegermann, M. Plato, B. V. Maltzan, K. Möbius, Mol. Phys. 1993, 78, 10571074.

[119] C. Corvaja, B. Kozankiewicz, L. Montanari, J. Prochorow, Chem. Phys. 1990, 142, 8390.

[120] M. Yagi, Chem. Phys. Lett. 1986, 124, 459462. 